\title{
Premature Coronary Artery Disease Is More Prevalent in People Who Go to Bed Late
}

\author{
Farzad Masoudkabir, MD, MPH ${ }^{1,2 *}$; Zahra Mohammadi, PhD ${ }^{3 *}$; Mohammad Alirezaei, MD ${ }^{4}$; Bahman Cheraghian, PhD ${ }^{5}$; Zahra \\ Rahimi, PhD Student ${ }^{6}$; Pegah Roayaei, MD; Mohammad Reza Naderian, MD; Leila Danehchin, MD ${ }^{8}$; Yousef Paridar, MD"; Farhad \\ Abolnezhadian, MD ${ }^{10,11}$; Ali Vasheghani-Farahani, MD ${ }^{1,2}$; Mohammad Noori, MD ${ }^{12}$; Seyed Ali Mard, MD ${ }^{13}$; Sahar Masoudi, MSc ${ }^{3}$; \\ Ali Akbar Shayesteh, MD ${ }^{13^{*}}$; Hossein Poustchi, MD, PhD ${ }^{3^{*}}$ \\ 'Cardiac Primary Prevention Research Center, Cardiovascular Diseases Research Institute, Tehran University of Medical Sciences, \\ Tehran, Iran \\ ${ }^{2}$ Department of Cardiology, Tehran Heart Center, Tehran University of Medical Sciences, Tehran, Iran \\ ${ }^{3}$ Liver and Pancreatobiliary Diseases Research Center, Digestive Diseases Research Institute, Tehran University of Medical Sciences, \\ Tehran, Iran \\ ${ }^{4}$ Students' Scientific Research Center, Tehran University of Medical Sciences, Tehran, Iran \\ ${ }^{5}$ Alimentary Tract Research Center, Clinical Sciences Research Institute, Department of Biostatistics and Epidemiology, School of \\ Public Health, Ahvaz Jundishapur University of Medical Sciences, Ahvaz, Iran \\ ${ }^{6}$ Department of Epidemiology, School of Public Health Iran University of Medical Sciences, Tehran, Iran \\ ${ }^{7}$ Non-Communicable Diseases Research Center, Endocrinology and Metabolism Population Sciences Institute, Tehran University of \\ Medical Sciences, Tehran, Iran \\ ${ }^{8}$ Behbahan Faculty of Medical Sciences, Behbahan, Iran \\ ${ }^{9}$ School of Medicine, Dezful University of Medical Sciences, Dezful, Iran \\ ${ }^{10}$ Shoushtar Faculty of Medical Sciences, Shoushtar, Iran \\ ${ }^{11}$ Ahvaz Jundishapur University of Medical Sciences, Ahvaz, Iran \\ ${ }^{12}$ Abadan Faculty of Medical Sciences, Abadan, Iran \\ ${ }^{13}$ Alimentary Tract Research center, Clinical sciences Research Institute, Ahvaz jundishapur University of Medical sciences, Ahvaz, Iran
}

\section{Abstract}

Background: Little is known regarding the impact of quantity and quality of sleep on the incidence of cardiovascular disease. The aim of this study was to investigate the possible independent association of late bedtime and premature coronary artery disease (PCAD).

Methods: Between October 2016 and November 2019, we conducted a cross-sectional population-based study on 30101 participants aged 20-65 years in Khuzestan Comprehensive Health Study (KCHS). Data on major risk factors of cardiovascular disease, habit history, physical activity, and sleep behavior was gathered and participants underwent blood pressure, anthropometric, and serum lipid and glucose profile measurements. PCAD was defined as documented history of developing obstructive coronary artery disease before 45 years in men and before 55 years in women.

Results: Of a total of 30101 participants (64.1\% female, mean age: $41.7 \pm 11.7$ years) included in this study, 1602 (5.3\%, 95\% confidence interval: $5.1 \%-5.6 \%)$ had PCAD. Late bedtime was reported in 7613 participants $(25.3 \%, 95 \%$ confidence interval: 24.9\%-25.8\%). Age-sex standardized prevalence for PCAD and late bedtime were 3.62 (3.43-3.82) and 27.8 (27.2-28.4), respectively. There was no significant difference $(P=0.558)$ regarding prevalence of PCAD between those with late bedtime $(5.5 \%, 95 \% \mathrm{Cl}: 4.9 \%-6.0 \%)$ and those with early bedtime $(5.3 \%, 95 \% \mathrm{Cl}: 5.0 \%-5.6 \%)$. However, after adjustment for potential confounders, late bedtime was independently associated with PCAD (OR=1.136, 95\% Cl=1.002-1.288, $P=0.046)$.

Conclusion: In this study, late bedtime was significantly associated with presence of PCAD. Future prospective studies should elucidate the exact role of late bedtime in developing coronary atherosclerosis prematurely.

Keywords: Coronary artery disease, Prevention, Sleep health

Cite this article as: Masoudkabir F, Mohammadi Z, Alirezaei M, Cheraghian B, Rahimi Z, Roayaei P, et al. Premature coronary artery disease is more prevalent in people who go to bed late. Arch Iran Med. 2021;24(12):876-880. doi: 10.34172/aim.2021.131

Received: September 15, 2020, Accepted: January 26, 2021, ePublished: December 1, 2021

\section{Introduction}

Ischemic heart disease is the leading cause of death ${ }^{1,2}$ and years of life lost ${ }^{3}$ world-wide. Although most of the coronary artery disease (CAD) occurs in the elderly, it has been reported that $4-10 \%$ of individuals with CAD are younger than 45 years. ${ }^{4}$ Despite numerous definitions and age cut-offs ${ }^{5,6}$ for premature coronary artery disease (PCAD), it is clear that developing CAD in younger ages has extra burden on societies because of involving people in their more productive years of life. This problem is more prominent in Middle-Eastern countries and particularly Iran where PCAD is more prevalent than Western countries. ${ }^{7}$ Hence, identifying the independent predictors of developing $\mathrm{CAD}$ at younger ages is of outmost importance to develop evidence-based preventive measures. ${ }^{7-10}$ 
There is evidence that sleep behavior, including sleep duration and bedtime, as an important part of lifestyle has changed in the last decades and it has been reported that people sleep shorter ${ }^{11,12}$ and go to bed later ${ }^{13}$ than before. It has led researchers to investigate the probable effects on sleep behavioral changes of different aspects of health. Studies have demonstrated that sleep duration is associated with obesity, insulin resistance, metabolic syndrome, hyperlipidemia, diabetes mellitus, and also CAD. ${ }^{14-19}$ Furthermore, bedtime, as an indicator for congruence of sleep and circadian rhythm, has a significant relationship with obesity, diabetes mellitus, and insulin resistance in childhood and adulthood. ${ }^{15,20-22}$ However, there is limited data regarding the possible association of bedtime in adults and presence of PCAD. Hence, we aimed to evaluate the relationship between late bedtime and PCAD in a large sample of participant in Khuzestan Comprehensive Health Study (KCHS).

\section{Materials and Methods Study Population}

This study is a subset of the KCHS, which was conducted with the aim of evaluating the health status of the people from the Khuzestan province. The province, with 27 counties and a population of 4909285 people, is located in southwestern Iran. KCHS was founded by the National Institute for Medical Research Development (NIMAD) in cooperation with the Digestive Diseases Research Institute (DDRI), Jundishapur Medical University, Iran Blood Transfusion Organization, and Abadan, Dezful and Behbahan Faculties.

\section{Sampling Method}

KCHS was performed in the period of October 2016 to November 2019 and the samples were selected using the multi-stage random sampling method. Consequently, 30101 individuals between 20-65 years of age who were residents of the Khuzestan province for at least one year were enrolled in the study. ${ }^{23}$

\section{Study Protocol}

Those who fulfilled all the inclusion criteria were invited face-to-face by trained staff and those who were unwilling to participate at any stage of the study for any reason, and individuals with mental, psychological or physical disabilities who were not able to complete the questioning process were excluded from the study. After a comprehensive review of the study procedures, each participant signed an informed consent.

Trained nurses performed blood pressure and anthropometric measurements. Height (in centimeters) and weight (in kilograms) were measured. Then, body mass index (BMI) was calculated by dividing weight (in kilograms) by height (in meters squared). Blood pressure was measured with Riester sphygmomanometers, twice on each arm in a standard sitting state. The lower reading on the arm with a higher systolic blood pressure was considered as the participant's blood pressure.

Using self-designed questionnaires, data on demographic characteristics, and past medical history, including history of CAD, traditional risk factors of cardiovascular disease, habit history, and physical activity were collected. Moreover, participants were asked about the time when they go to bed routinely.

After 8-12 hours of overnight fasting, a venous blood sample of 15 milliliters was collected from each participant. Afterward, levels of parameters, including fasting blood sugar (FBS), urea, creatinine (Cr), triglyceride (TG), total cholesterol (Chol), and high-density lipoproteins cholesterol (HDL) were measured in serum samples. ${ }^{22}$

\section{Definitions}

We defined late bedtime as bedtime after 1:00 AM. PCAD was defined as history of acute myocardial infarction (documented in hospital discharge summary) or obstructive CAD diagnosed by coronary angiography or computed tomography coronary angiography before 45 years in men and before 55 years in women.

\section{Statistical Analysis}

Mean \pm standard deviations were calculated for continuous variables and were compared between two independent groups using Student's t-test. Categorical variables were compared using the chi-square test or Fisher's exact test, as appropriate, and were presented as absolute frequencies with percentages. Multivariate logistic regression analysis was used to evaluate the independent predictors of PCAD. Two-tailed $P$ values were reported with significance defined at $P \leq 0.05$.

\section{Results}

Of a total of 30101 participants (64.1\% female, mean age: $41.7 \pm 11.7$ years) included in this study, 1602 (5.3\%, 95\% confidence interval: $5.1 \%-5.6 \%)$ had PCAD. Late bedtime was reported in 7613 participants $(25.3 \%$, 95\% confidence interval: $24.9 \%-25.8 \%)$. The age-sex-standardized prevalence rates for PCAD and late bedtime were 3.62 (3.43-3.82) and 27.8 (27.2-28.4), respectively. Table 1 demonstrates the demographic and clinical characteristics of the study population.

As shown in Table 1, participants who reported to go to bed late were younger and were less likely to have hypertension and positive family history of PCAD than those who reported to go to bed before 1:00 AM. Meanwhile, those who reported to go to bed late were more likely to smoke cigarettes, and have obesity defined as $\mathrm{BMI} \geq 30 \mathrm{~kg} / \mathrm{m}^{2}$ than those who reported to go to bed early.

There was no significant difference $(P=0.558)$ regarding the prevalence of $\mathrm{PCAD}$ between those with late bedtime $(5.5 \%, 95 \% \mathrm{CI}: 4.9 \%-6.0 \%)$ and those with early bedtime $(5.3 \%$, 95\% CI: 5.0\%-5.6\%). After adjustment for potential confounders including age, gender, diabetes mellitus, hypertension, cigarette smoking, family history 
Table 1. Demographic and Clinical Characteristics of the Study Population

\begin{tabular}{|c|c|c|c|}
\hline Characteristics & $\begin{array}{l}\text { Late Bedtime } \\
\qquad(\mathrm{n}=7613)\end{array}$ & $\begin{array}{l}\text { Early Bedtime } \\
\qquad(\mathrm{n}=22488)\end{array}$ & $P$ Value \\
\hline \multicolumn{4}{|l|}{ Age group (y), n (\%) } \\
\hline $20-34$ & $3022(39.8)$ & $6724(29.9)$ & $<0.0001$ \\
\hline $35-49$ & $2654(34.9)$ & $8950(39.8)$ & \\
\hline $50-65$ & $1922(25.3)$ & $6814(30.3)$ & \\
\hline Female, n (\%) & $4824(63.4 \%)$ & $14505(64.5 \%)$ & 0.073 \\
\hline $\mathrm{TCH} / \mathrm{HDL}$, mean (SD) & $3.96(1.17)$ & $3.93(1.20)$ & 0.045 \\
\hline Diabetes mellitus, n (\%) & $637(8.4)$ & $2015(9.0)$ & 0.100 \\
\hline HTN, n (\%) & $821(10.8)$ & $2653(11.8)$ & 0.015 \\
\hline BMI $\left(\mathrm{kg} / \mathrm{m}^{2}\right), \mathrm{n}(\%)$ & & & $<0.0001$ \\
\hline$<18.5$ & $213(2.8)$ & $607(2.7)$ & \\
\hline $18.5-24.9$ & $2046(27.3)$ & $6724(29.9)$ & \\
\hline $25.0-29.9$ & $2781(37.1)$ & $8500(37.8)$ & \\
\hline$\geq 30$ & $2457(32.8)$ & $6657(29.6)$ & \\
\hline Family history of CAD, $\mathrm{n}(\%)$ & $1419(18.6)$ & $4752(21.2)$ & $<0.0001$ \\
\hline Cigarette smoking, n (\%) & $931(12.2)$ & $2325(10.4)$ & $<0.0001$ \\
\hline Physical activity, n (\%) & & & $<0.0001$ \\
\hline Mild & $2448(32.2)$ & 6881(30.6) & \\
\hline Moderate & $3376(44.4)$ & $9535(42.4)$ & \\
\hline Intense & $1779(23.4)$ & $6072(27.0)$ & \\
\hline Premature CAD & $416(5.5)$ & $1186(5.3)$ & 0.558 \\
\hline
\end{tabular}

HTN, Hypertension; BMI, Body mass index; TCH, total cholesterol; HDL, high-density lipoprotein; $\mathrm{CAD}$, coronary artery disease. Continuous variables are presented as mean (SD) or median (25th, 75th percentile). Categorical variables are described as frequency (percentage).

of PCAD, physical activity and LDL/HDL ratio, late bedtime was independently associated with PCAD (odds ratio $[\mathrm{OR}]=1.136,95 \%$ confidence interval $=1.002-1.288$, $P=0.046$ ). Table 2 shows the independent predictors of PCAD in the study population.

\section{Discussion}

The main finding of our study was that late bedtime is independently associated with presence of PCAD. Despite numerous studies in children and adolescents evaluating the possible linkage of late bedtime and various aspects of health, ${ }^{24}$ there is lack of data regarding adulthood sleep behavior and cardiovascular diseases. To the best of our knowledge, this is the first study to report the independent association of late bedtime and PCAD.

Our findings confirms and expands the previous studies reporting a significant association between late bedtime in childhood and adolescence with major risk factors of CAD including obesity, ${ }^{25} \mathrm{BMI},{ }^{20,24,26}$ hypertension, ${ }^{27,28}$ reduced insulin sensitivity, ${ }^{15}$ and diabetes mellitus. ${ }^{22}$ Although late bedtime might predispose the person to PCAD via increased risk of abovementioned risk factors, we observed that even after adjustment for all abovementioned risk factors, late bedtime was independently associated with PCAD. This observation signals for other mechanistic pathways for association of late bedtime and PCAD. There is evidence that sleep deprivation and circadian rhythm
Table 2. Logistic Regression Analysis for Evaluation of Independent Predictors of Premature Coronary Artery Disease

\begin{tabular}{lccc}
\hline Variables & Odds Ratio & $\mathbf{9 5} \% \mathbf{C l}$ & $\boldsymbol{P}$ Value \\
\hline Age, year & 1.07 & $1.06-1.07$ & $<0.0001$ \\
Female & 0.82 & $0.72-0.94$ & 0.004 \\
Diabetes mellitus & 2.23 & $1.96-2.53$ & $<0.0001$ \\
\hline HTN & 3.50 & $3.10-3.39$ & $<0.0001$ \\
Body mass index, kg/m² & 1.02 & $1.01-1.03$ & $<0.0001$ \\
Family history of CAD & 2.04 & $1.81-2.29$ & $<0.0001$ \\
Cigarette smoking & 1.50 & $1.27-1.78$ & $<0.0001$ \\
Physical activity & & & 0.055 \\
\multicolumn{1}{c}{ Mild } & 1.15 & $0.99-1.34$ & \\
\multicolumn{1}{l}{ Moderate } & 1.00 & $0.87-1.16$ & \\
\multicolumn{1}{l}{ Intense } & 1 & & $<0.0001$ \\
\hline LDL/HDL & 0.87 & $0.82-0.92$ & 0.046 \\
\hline Bedtime after 1:00 AM & 1.13 & $1.00-1.28$ & \\
\hline Cl, Confidence interval; HTN, Hypertension; LDL, low-density lipoprotein; \\
HDL, high-density lipoprotein.
\end{tabular}

disruption may lead to oxidative stress in humans..$^{29,30}$ Sleep deprivation and restricted sleep times are also associated with increased plasma levels of proinflammatory cytokines including interleukin (IL)-6, IL-1 $\beta$, and tumor necrosis factor alpha (TNF- $\alpha)^{31-34}$ Irwin et al demonstrated that sleep loss induces spontaneous monocytic expression of IL- 6 and TNF- $\alpha$ and of signal transducer and activator of transcription (STAT) family proteins, which together map the dynamics of sleep loss on the molecular signaling pathways that regulate inflammatory and other immune responses. ${ }^{35}$ Given the pivotal role of oxidative stress and inflammation in initiation and progression of coronary atherosclerosis, ${ }^{2}$ it might be concluded that late bedtime contributes to PCAD through induction of inflammation and oxidative stress.

Although multiple studies have been performed on health impacts of late bedtime, there is no agreement regarding the definition of late bedtime and various studies have chosen different times. In contrast with our study, Yan et $\mathrm{a}^{22}$ and Sasaki et a ${ }^{36}$ chose going to bed after 12:00 AM as late bedtime. It seems reasonable to consider bedtime as a continuous variable and invoke receiver operating characteristics curve analysis for choosing an optimal cutoff time as late bedtime. Furthermore, it has been shown that systematic changes in the output of the human circadian pacemaker happen in the aging process ${ }^{37}$; so, allocating age-related cut-offs for future studies seems reasonable.

Our study has some limitations that should be mentioned. One of the limitations is that we evaluated sleep behavior through self-reported questionnaires. Studies that evaluated the correlation of objective and subjective sleep duration, have reported a weak ${ }^{20}$ to moderate correlation. ${ }^{38-40}$ Another limitation is that we did not evaluate some sleep relating factors, such as sleep quality and insomnia which can potentially confound the association of bedtime and PCAD. ${ }^{41}$ In this study, $64.1 \%$ of 
the participants were female while according to the latest census in 2016, only $49.3 \%$ of residents in the Khuzestan province had female sex. Although this is a common selection bias in studies with volunteer participation, its probable impact on results should be acknowledged. Finally, due to the cross-sectional design of the study, a causal relationship cannot be inferred. Large-scale prospective cohort studies should elucidate the exact role of sleep behavior on developing CAD prematurely.

In conclusion, our study demonstrated a significant independent association between going to bed after 1:00 $\mathrm{AM}$ and PCAD. This finding supports the importance of bedtime in health. Future prospective studies should examine the possible causal relationship of late bedtime and PCAD.

\section{Authors' Contribution}

FMK, HP, ZM, and AAS designed and performed project and drafted the paper. MA, BC, ZR, LD, YP, FA, MN, and SAM contributed to collect data and drafted the paper. MRN, PR, AVF, and SM analyzed data and drafted the paper.

\section{Conflict of Interest Disclosures}

The authors declare that there is no conflict of interest.

\section{Ethical Statement}

The ethics committee of NIMAD approved the protocol of KCHS at IR.NIMAD.REC.1394.002.

\section{Funding}

KCHS was supported by a grant from NIMAD (grant number: 940406).

\section{References}

1. World Health Organization (WHO). The Top 10 Causes of Death. WHO; 2020. Available from: https://www.who.int/ news-room/fact-sheets/detail/the-top-10-causes-of-death.

2. Masoudkabir F, Sarrafzadegan N, Gotay C, Ignaszewski A, Krahn AD, Davis MK, et al. Cardiovascular disease and cancer: evidence for shared disease pathways and pharmacologic prevention. Atherosclerosis. 2017;263:343-51. doi: 10.1016/j. atherosclerosis.2017.06.001.

3. Wang H, Naghavi M, Allen C, Barber RM, Bhutta ZA, Carter $A$, et al. Global, regional, and national life expectancy, allcause mortality, and cause-specific mortality for 249 causes of death, 1980-2015: a systematic analysis for the Global Burden of Disease Study 2015. Lancet. 2016;388(10053):1459-544. doi: 10.1016/s0140-6736(16)31012-1.

4. Mohammad AM, Jehangeer HI, Shaikhow SK. Prevalence and risk factors of premature coronary artery disease in patients undergoing coronary angiography in Kurdistan, Iraq. BMC Cardiovasc Disord. 2015;15:155. doi: 10.1186/s12872-0150145-7.

5. Panwar RB, Gupta R, Gupta BK, Raja S, Vaishnav J, Khatri $M$, et al. Atherothrombotic risk factors \& premature coronary heart disease in India: a case-control study. Indian J Med Res. 2011;134(1):26-32.

6. Khot UN, Khot MB, Bajzer CT, Sapp SK, Ohman EM, Brener $\mathrm{S}$, et al. Prevalence of conventional risk factors in patients with coronary heart disease. JAMA. 2003;290(7):898-904. doi: 10.1001/jama.290.7.898.

7. Poorzand H, Tsarouhas K, Hozhabrossadati SA, Khorrampazhouh N, Bondarsahebi Y, Bacopoulou F, et al. Risk factors of premature coronary artery disease in Iran: a systematic review and meta-analysis. Eur J Clin Invest. 2019;49(7):e13124. doi: 10.1111/eci.13124.

8. Hoo FK, Foo YL, Lim SM, Ching SM, Boo YL. Acute coronary syndrome in young adults from a Malaysian tertiary care centre. Pak J Med Sci. 2016;32(4):841-5. doi: 10.12669/ pjms.324.9689.

9. Sawhney JPS, Prasad SR, Sharma M, Madan K, Mohanty A, Passey R, et al. Prevalence of familial hypercholesterolemia in premature coronary artery disease patients admitted to a tertiary care hospital in North India. Indian Heart J. 2019;71(2):118-22. doi: 10.1016/j.ihj.2018.12.004.

10. Setia N, Verma IC, Khan B, Arora A. Premature coronary artery disease and familial hypercholesterolemia: need for early diagnosis and cascade screening in the Indian population. Cardiol Res Pract. 2012;2012:658526. doi: 10.1155/2012/658526.

11. Ford ES, Cunningham TJ, Croft JB. Trends in self-reported sleep duration among US adults from 1985 to 2012. Sleep. 2015;38(5):829-32. doi: 10.5665/sleep.4684.

12. Sheehan CM, Frochen SE, Walsemann KM, Ailshire JA. Are U.S. adults reporting less sleep?: findings from sleep duration trends in the National Health Interview Survey, 2004-2017. Sleep. 2019;42(2):zsy221. doi: 10.1093/sleep/zsy221.

13. Norell-Clarke A, Hagquist C. Changes in sleep habits between 1985 and 2013 among children and adolescents in Sweden. Scand J Public Health. 2017;45(8):869-77. doi: 10.1177/1403494817732269.

14. Wu Y, Zhai L, Zhang D. Sleep duration and obesity among adults: a meta-analysis of prospective studies. Sleep Med. 2014;15(12):1456-62. doi: 10.1016/j.sleep.2014.07.018.

15. Simon SL, Behn CD, Cree-Green M, Kaar JL, Pyle L, Hawkins SMM, et al. Too late and not enough: school year sleep duration, timing, and circadian misalignment are associated with reduced insulin sensitivity in adolescents with overweight/obesity. J Pediatr. 2019;205:257-64.e1. doi: 10.1016/j.jpeds.2018.10.027

16. Xi B, He D, Zhang M, Xue J, Zhou D. Short sleep duration predicts risk of metabolic syndrome: a systematic review and meta-analysis. Sleep Med Rev. 2014;18(4):293-7. doi: 10.1016/j.smrv.2013.06.001.

17. Grandner MA, Chakravorty S, Perlis ML, Oliver L, Gurubhagavatula I. Habitual sleep duration associated with self-reported and objectively determined cardiometabolic risk factors. Sleep Med. 2014;15(1):42-50. doi: 10.1016/j. sleep.2013.09.012.

18. Wang D, Li W, Cui X, Meng Y, Zhou M, Xiao L, et al. Sleep duration and risk of coronary heart disease: a systematic review and meta-analysis of prospective cohort studies. Int J Cardiol. 2016;219:231-9. doi: 10.1016/j.ijcard.2016.06.027.

19. Lao XQ, Liu X, Deng HB, Chan TC, Ho KF, Wang F, et al. Sleep quality, sleep duration, and the risk of coronary heart disease: a prospective cohort study with 60,586 adults. J Clin Sleep Med. 2018;14(1):109-17. doi: 10.5664/jcsm.6894.

20. Lee S, Hale L, Chang AM, Nahmod NG, Master L, Berger LM, et al. Longitudinal associations of childhood bedtime and sleep routines with adolescent body mass index. Sleep. 2019;42(1):zsy202. doi: 10.1093/sleep/zsy202.

21. Anderson SE, Andridge R, Whitaker RC. Bedtime in preschoolaged children and risk for adolescent obesity. J Pediatr. 2016;176:17-22. doi: 10.1016/j.jpeds.2016.06.005.

22. Yan B, Fan Y, Zhao B, He X, Yang J, Chen C, et al. Association between late bedtime and diabetes mellitus: a large community-based study. J Clin Sleep Med. 2019;15(11):16217. doi: 10.5664/jcsm.8026.

23. Cheraghian B, Sharafkhah M, Mohammadi Z, Hariri S, Rahimi Z, Danehchin L, et al. The Khuzestan Comprehensive Health Study (KCHS): methodology and profile of participants. Arch Iran Med. 2020;23(10):653-7. doi: 10.34172/aim.2020.82. 
24. Asarnow LD, McGlinchey E, Harvey AG. Evidence for a possible link between bedtime and change in body mass index. Sleep. 2015;38(10):1523-7. doi: 10.5665/sleep.5038.

25. Liu S, Zhang J, Ma J, Shang Y, Ma Y, Zhang X, et al. Synergistic interaction between bedtime and eating speed in predicting overweight and obesity in Chinese preschool-aged children. Aging (Albany NY). 2019;11(7):2127-37. doi: 10.18632/ aging.101906.

26. Golley RK, Maher CA, Matricciani L, Olds TS. Sleep duration or bedtime? exploring the association between sleep timing behaviour, diet and BMI in children and adolescents. Int J Obes (Lond). 2013;37(4):546-51. doi: 10.1038/ijo.2012.212.

27. Jansen EC, Dunietz GL, Matos-Moreno A, Solano M, LazcanoPonce E, Sánchez-Zamorano LM. Bedtimes and blood pressure: a prospective cohort study of Mexican adolescents. Am J Hypertens. 2020;33(3):269-77. doi: 10.1093/ajh/ hpz191.

28. Mi SJ, Kelly NR, Brychta RJ, Grammer AC, Jaramillo M, Chen KY, et al. Associations of sleep patterns with metabolic syndrome indices, body composition, and energy intake in children and adolescents. Pediatr Obes. 2019;14(6):e12507. doi: 10.1111/ijpo.12507.

29. Nagata C, Tamura T, Wada K, Konishi K, Goto Y, Nagao Y, et al. Sleep duration, nightshift work, and the timing of meals and urinary levels of 8-isoprostane and 6-sulfatoxymelatonin in Japanese women. Chronobiol Int. 2017;34(9):1187-96. doi: 10.1080/07420528.2017.1355313.

30. Tobaldini E, Costantino G, Solbiati M, Cogliati C, Kara T, Nobili L, et al. Sleep, sleep deprivation, autonomic nervous system and cardiovascular diseases. Neurosci Biobehav Rev. 2017;74(Pt B):321-9. doi: 10.1016/j.neubiorev.2016.07.004.

31. Ferrie JE, Kivimäki $M$, Akbaraly TN, Singh-Manoux A, Miller MA, Gimeno D, et al. Associations between change in sleep duration and inflammation: findings on $\mathrm{C}$-reactive protein and interleukin 6 in the Whitehall II Study. Am J Epidemiol. 2013;178(6):956-61. doi: 10.1093/aje/kwt072.

32. Haack M, Sanchez E, Mullington JM. Elevated inflammatory markers in response to prolonged sleep restriction are associated with increased pain experience in healthy volunteers. Sleep. 2007;30(9):1145-52. doi: 10.1093/ sleep/30.9.1145.
33. van Leeuwen $W M$, Lehto $M$, Karisola $P$, Lindholm $H$, Luukkonen R, Sallinen $M$, et al. Sleep restriction increases the risk of developing cardiovascular diseases by augmenting proinflammatory responses through IL-17 and CRP. PLoS One. 2009;4(2):e4589. doi: 10.1371/journal.pone.0004589.

34. Vgontzas AN, Zoumakis E, Bixler EO, Lin HM, Follett $H$, Kales $A$, et al. Adverse effects of modest sleep restriction on sleepiness, performance, and inflammatory cytokines. J Clin Endocrinol Metab. 2004;89(5):2119-26. doi: 10.1210/ jc.2003-031562.

35. Irwin MR, Witarama T, Caudill M, Olmstead R, Breen EC. Sleep loss activates cellular inflammation and signal transducer and activator of transcription (STAT) family proteins in humans. Brain Behav Immun. 2015;47:86-92. doi: 10.1016/j. bbi.2014.09.017.

36. Sasaki N, Fujiwara S, Yamashita H, Ozono R, Monzen $\mathrm{Y}$, Teramen $\mathrm{K}$, et al. Association between obesity and selfreported sleep duration variability, sleep timing, and age in the Japanese population. Obes Res Clin Pract. 2018;12(2):187-94. doi: 10.1016/j.orcp.2017.10.008.

37. Czeisler CA, Dumont M, Duffy JF, Steinberg JD, Richardson GS, Brown EN, et al. Association of sleep-wake habits in older people with changes in output of circadian pacemaker. Lancet. 1992;340(8825):933-6. doi: 10.1016/0140-6736(92)92817-y.

38. Jackson CL, Ward JB, Johnson DA, Sims M, Wilson J, Redline S. Concordance between self-reported and actigraphy-assessed sleep duration among African-American adults: findings from the Jackson Heart Sleep Study. Sleep. 2020;43(3):zsz246. doi: 10.1093/sleep/zsz246.

39. Silva GE, Goodwin JL, Sherrill DL, Arnold JL, Bootzin RR, Smith T, et al. Relationship between reported and measured sleep times: the sleep heart health study (SHHS). J Clin Sleep Med. 2007;3(6):622-30.

40. Girschik J, Fritschi L, Heyworth J, Waters F. Validation of selfreported sleep against actigraphy. J Epidemiol. 2012;22(5):4628. doi: 10.2188/jea.je20120012.

41. Hoevenaar-Blom MP, Spijkerman AM, Kromhout D, van den Berg JF, Verschuren WM. Sleep duration and sleep quality in relation to 12-year cardiovascular disease incidence: the MORGEN study. Sleep. 2011;34(11):1487-92. doi: 10.5665/ sleep.1382. 